\title{
ASO Author Reflections: Standardizing HIPEC Methodology and Regimens: a Prelude to the PSOGI Expert Consensus
}

\author{
Aditi Bhatt, MS, $\mathrm{MCh}^{1}$, Kurt Van Der Speeten, $\mathrm{MD}, \mathrm{PhD}^{2}$, Martin Hubner, $\mathrm{MD}, \mathrm{PhD}^{3}$, Shigeki Kusamura, $\mathrm{MD}$, \\ $\mathrm{PhD}^{4}$, and Olivier Glehen, $\mathrm{MD}, \mathrm{PhD}^{5}$ \\ ${ }^{1}$ Department of Surgical Oncology, Zydus Hospital, Ahmedabad, India; ${ }^{2}$ Department of Surgical Oncology, Zeikenhuis \\ Oost-Limberg, Genk, Belgium; ${ }^{3}$ Department of Visceral Surgery, Lausanne University Hospital CHUV, Lausanne, \\ Switzerland; ${ }^{4}$ Department of Surgical Oncology, Fondazione IRCCS Istituto Nazionale dei Tumori, Milan, Italy; \\ ${ }^{5}$ Department of Surgical Oncology, Centre Hospitalier Lyon-Sud, Hospices Civils de Lyon, Lyon, Pierre Bénite, France
}

\section{PAST}

Hyperthermic intraperitoneal chemotherapy (HIPEC) was developed in combination with cytoreductive surgery (CRS) for the treatment of peritoneal metastases (PMs) from different primary tumors in an era when hyperthermia was being used extensively in the treatment of different malignancies. Systemic chemotherapy (SC) produced dismal results for most tumors. The understanding of the biology of PMs has grown, along with the experience with HIPEC, which has moved rapidly from the bench to the bedside. ${ }^{1}$ The initial decades were spent in developing centers of expertise to reduce the proportion of incomplete resections and the morbidity and mortality from the procedure. Due to gaps in the preclinical data and clinical experience, parameters for evaluating efficacy were poorly defined and efficacy was evaluated for the combination of CRS and HIPEC. During this period, surgeons developed a multitude of HIPEC regimens using different drug combinations with multiple variations in the methodology and elements vital to the procedure. ${ }^{2}$ Some recent randomized trials have not shown a benefit of HIPEC over CRS alone and the question has been raised as to whether a different regimen would produce a favorable outcome.

(C) Society of Surgical Oncology 2021

First Received: 10 May 2021

Accepted: 10 May 2021;

Published Online: 4 June 2021

O. Glehen, $\mathrm{MD}, \mathrm{PhD}$

e-mail: olivier.glehen@chu-lyon.fr

\section{PRESENT}

The use of newer SC regimens and targeted therapies has improved the survival in patients with PMs treated with systemic therapies alone, and SC is an essential adjunct to CRS and HIPEC for most tumors. At the same time, a multitude of clinical trials are being conducted to evaluate the benefit of HIPEC for different indications. It is important to determine which regimens are best suited for evaluation in clinical trials and which regimens should be used for indications where there are no data from randomized trials. There is a need for peritoneal malignancy centers across the world to adopt a limited number of regimens. This will increase the collective experience with each regimen, make comparisons between studies more meaningful, and lead to a greater acceptance of the results of randomized trials.

Based on a critical review of the literature, we found that the preclinical/clinical data for most HIPEC regimens was limited and studies comparing different regimens were scarce. ${ }^{3}$ Many regimens were neither supported by preclinical rationale data nor validated by a dose escalating formal phase I trial. All regimens were based on pharmacokinetic data and did not take chemosensitivity of PMs into account.

\section{FUTURE}

More research comprising of preclinical and phase I/II studies and studies on biological mechanisms underlying the drug-heat interaction and cytotoxicity of chemotherapeutic agents needs to be conducted. However, not all parameters can be tested in randomized trials and the 
methodology and regimens need to be standardized until better evidence is available. The best way to minimize the heterogeneity is by carrying out an expert consensus that aims to identify and define a limited number of regimens for each indication and primary site. The Grading of Recommendations Assessment, Development, and Evaluation (GRADE) system for rating quality of evidence should be used to rate the evidence for such a consensus, especially since the evidence is limited. ${ }^{4}$ Regimens not supported by preclinical rationale data and not validated by a dose-escalating formal phase I trial should be eliminated. The choice of regimen can then be tailored to the patient profile and expected toxicity, as well as the methodology according to regional factors.

Surgeons should focus on methodological accuracy, as well as on the three key elements, i.e. adequate drug dosing, maintenance of hyperthermia, and adequate perfusion.

Personalized medicine approaches such as patient tumor organoids are being developed to determine the best regimen for each patient, but clinical validation is likely to be challenging. ${ }^{5}$

FUNDING The authors received no funding for this study.

DISCLOSURES Olivier Glehen has received honoraria from Gamida Tech. Aditi Bhatt, Kurt Van Der Speeten, Martin Hubner, and Shigeki Kusamura have no disclosures to declare.

\section{REFERENCES}

1. Stein MK, Williard FW, Xiu J, Tsao MW, Martin MG, Deschner $\mathrm{BW}$, et al. Comprehensive tumor profiling reveals unique molecular differences between peritoneal metastases and primary colorectal adenocarcinoma. J Surg Oncol. 2020;121(8):1320-8. h ttps://doi.org/10.1002/jso.25899.

2. Lemoine L, Sugarbaker P, Van der Speeten K. Drugs, doses, and durations of intraperitoneal chemotherapy: standardising HIPEC and EPIC for colorectal, appendiceal, gastric, ovarian peritoneal surface malignancies and peritoneal mesothelioma. Int J Hyperth. 2017;33(5):582-92. ～https://doi.org/10.1080/02656736.2017. 1291999.

3. Bhatt A, de Hingh I, Van Der Speeten K, Hubner M, Deraco M, Bakrin N, et al. HIPEC methodology and regimens: the need for an expert consensus. Ann. Surg. Oncol. 2021. https://doi.org/10.1245/ s10434-021-10193-w.

4. Atkins D, Best D, Briss PA, Eccles M, Falck-Ytter Y, Flottorp S, et al. Grading quality of evidence and strength of recommendations. BMJ. 2004;328(7454):1490. https://doi.org/10.1136/bmj. 328.7454.1490.

5. Votanopoulos KI, Mazzocchi A, Sivakumar H, et al. Appendiceal cancer patient-specific tumor organoid model for predicting chemotherapy efficacy prior to initiation of treatment: a feasibility study. Ann Surg Oncol. 2019;26:139-47. https://doi.org/10.1245/ s10434-018-7008-2.

Publisher's Note Springer Nature remains neutral with regard to jurisdictional claims in published maps and institutional affiliations. 